\title{
Transport of Intensity Phase Reconstruction Revisited
}

\author{
A. Lubk $^{1,2}$, L. Clark ${ }^{2}$, G. Guzzinati ${ }^{2}$ and J. Verbeeck ${ }^{2}$ \\ ${ }^{1}$ Triebenberg Laboratory, Institute of Structure Physics, Technische Universität Dresden, 01062 Dresden, \\ Germany \\ ${ }^{2}$ EMAT, University of Antwerp, Groenenborgerlaan 171, 2020 Antwerp, Belgium
}

The phase problem can be considered as one of the cornerstones of quantum mechanics intimately connected to detection process and particle-wave duality. A large number of phase reconstruction schemes have been devised to date which exploit interference of waves with different phasing in one or the other way. Most notably off-axis holography uses a tilted references wave, whereas Gabor's original in-line scheme superimposes the wave field with itself by defocusing. The Transport of Intensity Equation (TIE) Reconstruction ${ }^{[1]}$ based on evaluating

$$
\frac{\partial \rho(\mathbf{r})}{\partial z}=-\frac{1}{k} \nabla_{\perp}\left(\rho(\mathbf{r}) \nabla_{\perp} \varphi(\mathbf{r})\right)
$$

in the paraxial regime can be considered as an infinitesimal version of the latter. Here $k$ is the wave vector, $\rho$ is the recorded density, $\partial \rho / \partial z$ is approximated by at least two slightly defocussed images and $\varphi$ is the unknown phase. It shares the advantage to not rely on off-axis reference waves and removes problems connected to nonlinearities occurring in the finite defocus in-line reconstruction (e.g. twin image, non-uniqueness).

Mathematically, the TIE is a linear elliptic partial differential equation for the phase $\varphi$ with a welldeveloped theory. For instance, uniqueness and existence of weak solutions on simply connected domains with $\rho>0$ is guaranteed by the Lax-Milgram theorem. However, wave fields frequently contain $\rho=0$. Here, the phase is not defined and the partial differential equation becomes singular, which requires that these loci have to be removed from the domain of the TIE. That includes defining boundary conditions (BCs) at the border of the excluded regions. The treatment of $\mathrm{BC}$ at the border of the field of view and at interior points where $\rho=0$ was insufficient in the past; it considered only a small subset of possible BCs (i.e. mainly periodic) and excluded or insufficiently treated points where $\rho=0$, which reduced the scope of the TIE reconstruction scheme. For instance phase singularities, attracting a lot of interest in photonics and electron optics, could not be treated with TIE.

Here we present a rigorous treatment of Eq. (1) removing these limitations. We show that the main problem consists of identifying $\rho=0$ loci and defining and limiting the range of BCs. We propose particular boundary conditions yielding a set of acceptable solutions, which are further refined to a unique solution by a consistency check with a strongly defocused image. We furthermore discuss the influence of partial coherence and successfully apply the method to reconstruct the phase of an experimental electron vortex beam with a central phase singularity $(\varphi=m \cdot \operatorname{atan} 2(y, x))$ as a proof of concept. The implementation of the method contains various steps ranging from recording and preprocessing a defocus series, defining $\mathrm{BCs}$, solving (1) with finite elements, and performing a consistency check with a strongly defocused image in the last step. An example is illustrated in Figure 1. 


\section{References:}

[1] Teague, M. R., J. Opt. Soc. Am., OSA, 1983, 73, 1434-1441

[2] The research leading to these results has received funding from the European Union Seventh Framework Program under Grant Agreement 312483 - ESTEEM2 (Integrated Infrastructure Initiative I3).

\section{Defocus series}

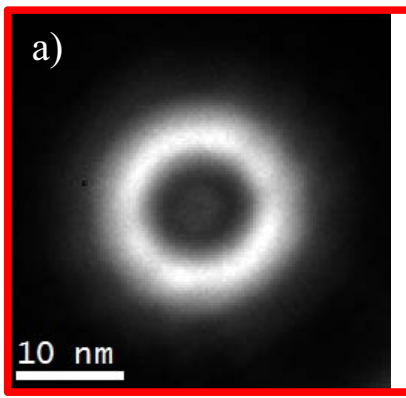

$z=-17 \mu \mathrm{m}$

$\mathrm{m}=1$ phase

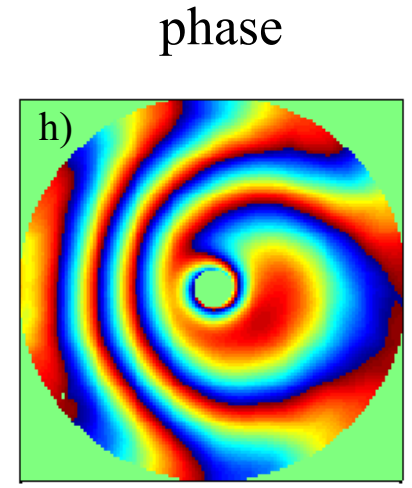

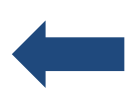

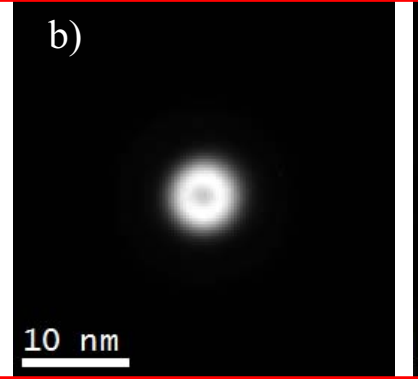

$z=0 \mu \mathrm{m}$
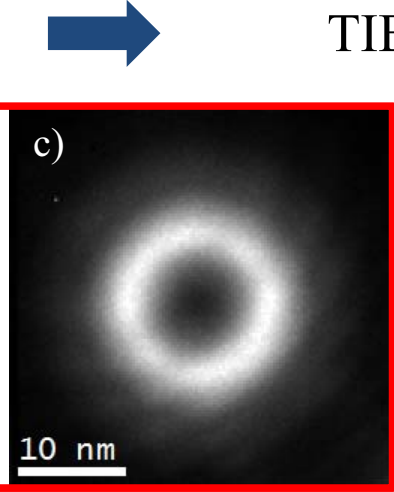

$z=17 \mu \mathrm{m}$

\section{TIE with different BCs}

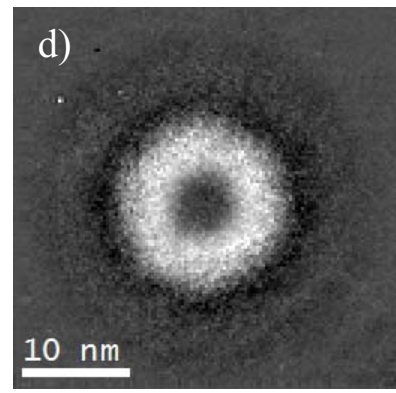

$\partial \rho / \partial z$

Consistency check at $\mathrm{z}=-17 \mu \mathrm{m}$

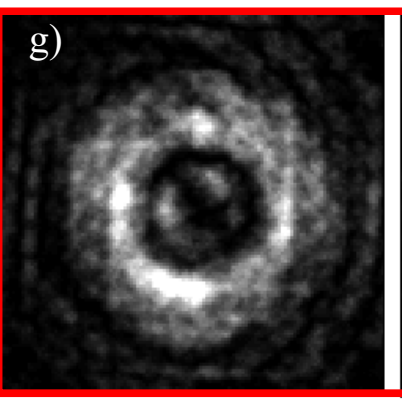

$\mathrm{m}=2$

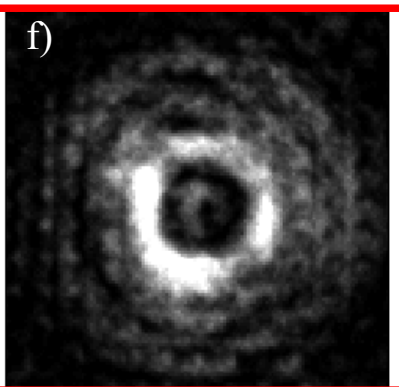

$\mathrm{m}=1$

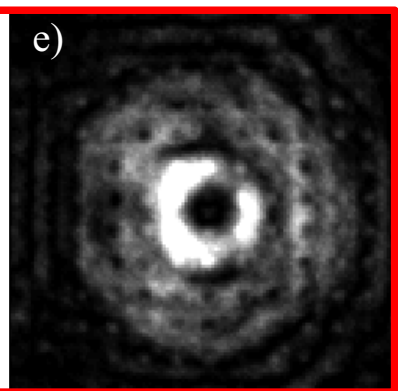

$\mathrm{m}=0$

Figure 1. TIE reconstruction scheme: a)-c) images from a defocus series. d) Density derivative approximated from two slightly defocused images, e)-g) Defocused solutions to $m=0,1,2$ vortices with $m=1$ fitting best to the experimental image a). h) Reconstructed phase of $m=1$ solution. The vorticity is concealed by the Hue colour space employed. The visible phase ramp is a consequence of the holographic diffraction grating used to produce the vortex beam. 